\title{
The Effects of Visual Biofeedback Balance Training on Functional Ability in Children with Cerebral Palsy : A Pilot Study
}

\author{
Chang-Kyo Yun $\cdot \mathrm{Ji}-\mathrm{Na}$ Yoo ${ }^{1 \dagger}$ \\ Department of Physical Therapy, College of Rehabilitation Science, Graduate School, Daegu University \\ ${ }^{1}$ Rehabilitation Medicine, The Joeun Hospital
}

Received: August 2, 2016 / Revised: August 2, 2016 / Accepted: August 5, 2016

(C) 2016 J Korean Soc Phys Med

\begin{abstract}
| Abstract |
PURPOSE: The purpose of this study is to examine the impact of balance training on a three-dimensional balance trainer that provides the up-and-down vertical movement of the knee jointand left-and-right horizontal movement, along with visual feedback on the functional ability of children with spastic cerebral palsy (CPs)
\end{abstract}

METHODS: 8 CPs participated in this study. The experiment was implemented for 40 minutes, three times a week for a total of six weeks. The subjects received general physiotherapy for 15 minutes in each session focused on balance and walking, as based on the neuro-developmental treatment theory. Balance training was performed for 20 minutes on a three-dimensional balance trainer where knee joint movement providing visual feedback is applied. The evaluations were conducted before and after the test, and posture sway was measured using 10 Meter Walking Test (10MWT), Timed Up \& Go Test (TUG), and the Good Balance System to evaluate the functional ability and balance

†Corresponding Author : vjinav0122@hanmail.net

This is an Open Access article distributed under the terms of the Creative Commons Attribution Non-Commercial License (http://creativecommons.org/licenses/by-nc/3.0) which permits unrestricted non-commercial use, distribution, and reproduction in any medium, provided the original work is properly cited. of the subjects

RESULTS: 10MWT was not statistically significant ( $>$.05). On the contrary, TUG and postural sway indicate static balance showed a statistically significant decrease $(\mathrm{p}<.05)$.

In a static balance test using the Good Balance System, the average moving speedstatistically significantly decreased in the $\mathrm{AP}$ and $\mathrm{ML}$ directions $(\mathrm{p}<.05)$, and the mean velocity moment also significantly decreased $(\mathrm{p}<.05)$.

CONCLUSION: These findings suggested that balance training using the three-dimensional balance trainer, with the features of visual feedback and up-and-down knee joint movement effects on increasing dynamic and static balance.

Key Words: Balance training, Visual feedback, Cerebral palsy

\section{Introduction}

Cerebral palsy is a non-progressive brain legion that induces diverse motor defects due to a convulsion or defect in the brain that develops before, during, or after birth (Nelson, 2003). Children with cerebral palsy not only have defects in the motor system, including abnormal muscle 
strength in the limbs, contracture, myotonus abnormality, inadequate timing, and decreased amplitude in muscle activation, but they also have defects in the visual, tactual, and proprioceptive senses, as well as in the vestibular systems. These defects cause difficulties in motor control and induce a loss of balance ability in children with cerebral palsy, who maintain posture through anticipatory postural adjustments, reactive postural adjustments (Bax et al., 2007; Bigongiari et al., 2011; Girolami et al., 2011). The loss of balance ability is a clinically important problem for children with cerebral palsy and it becomes an important factor regarding improving quality of life through daily living activities (Carlberg and Hadders-Algra, 2005; Van Der Heide et al., 2004). Moreover, balance ability acts as an important factor in relation to motor ability and work performance, which are required of children with cerebral palsy. Moreover, as it is the fundamental element of all motor ability, improvement of balance ability is critical in the treatment of children with cerebral palsy (Ferdjallah et al., 2002; Imms, 2008).

Previous studies mainly focused on training that improves the balance ability of children with cerebral palsy. They applied Neurodevelopmental Therapy (NDT), hippotherapy, treadmill training without weight bearing, body trunk muscle strengthening training, reactive balance training using a moving platform, visual feedback training, and others. However, the results were controversial (Shumway-Cook et al., 2003; Butler and Darrah, 2001; Dewar et al., 2015; Lee and Kim, 2011). Among these training methods, balance training using visual feedback aimed to correct the maintenance of the body on a vertical line, decrease the difference between the left and right body weight loads, and effectively increase the body's stability range (Duarte and Zatsiorsky, 2002; Chen et al., 2002; Ballaz et al., 2014; Sackley and Lincoln, 1997; Lee et al., 2015). However, these balance-training approaches mainly decrease postural sway on a two-dimensional horizontal plane and they have limitations in balance training on a vertical line, such as sitting and standing, which are critical in daily living movements. Besides, most previous studies involve patients who have suffered a stroke or aged people (Lee, 2013). As such, research on children with cerebral palsy is scarce as of now.

A three-dimensional balance trainer provides balance training using visual feedback through a monitor. It simultaneously induces the left-and-right horizontal movement and up-and-down vertical movement of the knee joint to stimulate the vestibular and proprioceptive senses at the same time for balance ability training. A safety bar and sling are installed on the side to provide a safer intervention to patients who have a high risk of falling. Moreover, the three-dimensional balance trainer provides a program that is more effective for balance training, as users learn about movements by solving problems based on the motor learning theory by Shumway-Cook, instead of the repeated performance of movement (Shumway-Cook and Horak, 1986; Byun et al., 2012). Hence, the purpose of this study is to examine the impact of balance training on a three-dimensional balance trainer that provides movement of the knee joint, along with visual feedback on the functional ability of children with spastic cerebral palsy.

\section{Methods}

This study received permission from theDaegu University Bioethics Committee and the research period was from April to June in 2015. The purpose and procedure of this study were explained to all of the research participants and their guardians in verbal and written form and the researcher received their consent. The subjects that participated in this study include 10 outpatients in Clinic D located in Daegu, Korea who were diagnosed with cerebral palsy (four girls and six boys). The selection criteria of the research subjects included children diagnosed 
cerebral palsy who have Gross Motor Function Classification System (GMFCS) I-III and a level of stiffness at Modified Ashworth Scale (MAS) under two. The subjects had sufficient cognitive ability to understand the test procedure and the researcher's direction. Moreover, the subjects had no problem in visual and auditory senses, as well as no experience of orthopedic surgery or botuline toxin treatment in the past six months.

The experiment was implemented for 40 minutes, three times a week for a total of six weeks. The subjects received general physiotherapy for 15 minutes in each session focused on balance and walking, as based on the neuro-developmental treatment theory. After a five-minute rest, balance training was performed for 20 minutes on a three-dimensional balance trainer where knee joint movement providing visual feedback is applied. The three-dimensional balance trainer is composed of a body wherein a game program and monitor are installed. The program processes information received from a pressure sensor on the force platform that can detect the left-and-right movement of the weight load and a tilt sensor that can detect the angle of the knee joint. The pressure sensor detects the center of weight of the patient and a cursor on a screen moves according to the left-and-right movement of the body's weight to provide visual feedback. The tilt sensor of the knee has a cursor that moves upward when folding the knees and downward when bending the knees to provide visual feedback. The game program that provides visual feedback is composed of sunflower planting that induces the left-and-right movement of the center of the body and Adventures of Sinbad and fruit grabbing, which induce both the left-and-right and up-and-down movements. For balance training, sunflower planting and Adventures of Sinbad were performed each for five minutes, and fruit grabbing was performed for ten minutes to maintain the interest and focus of the children with cerebral palsy. Before starting the game, the difficulty of the game was determined for each subject by evaluating their left-and-right weight distribution and folding and unfolding angles of the knees using the evaluation program. The difficulty was increased as skill increased.

All evaluations were performed by a physiotherapist who was unaware of the research purpose. The evaluations were conducted before and after the test, and posture sway was measured using 10MWT, Timed Up \& Go Test (TUG), and the Good Balance System ${ }^{\circledR}$ to evaluate the functional ability and balance of the subjects. Each measurement was conducted three times and the average value was used.

10MWT is a method that evaluates gait speed in a short period. The starting and ending points of a 14-meter route were marked. To measure the normal gait speeds of the subjects, time taken to walk 10 meters was measured after excluding the initial two-meter acceleration interval and the final two-meter deceleration interval.

TUG is an evaluation method that assesses the balance, movement, and mobility of aged people who have diminished balance ability or disabled children. The test measures the time spent from standing at a signal by a chair with a back and no armrest to walk around an obstacle at a three-meter distance and sit back down as fast as possible. The intra-rater reliability $(\mathrm{ICC}=.99)$ and inter-rater reliability ( $\mathrm{ICC}=.98)$ of the TUG test are reported to be very high (Podsiadlo and Richardson, 1991).

As for the postural sway of the subjects, it indicates static balance ability, as measured using the Good Balance System ${ }^{\circledR}$. The subjects were told to comfortably stand on the triangular force platform of the Good Balance System $\mathbb{R}$ with their feet shoulder-width apart for 30 seconds while looking at a monitor. For postural sway measurement, the subjects maintained a comfortable upright position when standing and they maintained bare feet for the measurement. Before starting the measurement, the measurer gave the subjects an oral direction of "please stand in the most comfortable way without movement." The Good Balance System ${ }^{\circledR}$ uses a computer to compute the average moving speed of Center of Pressure (COP) within Antero-Posterior 
(AP) $(\mathrm{mm} / \mathrm{s})$ and the average moving speed of COP, as well as the mean velocity moment value within the Medio-Lateral (ML) direction, and it displays the results (Ha et al., 2014).

All data were computed and were analyzed using the Statistical Package for Social Science (SPSS) version 20.0 for Windows. The mean and standard deviation were obtained using descriptive statistics, and a Wilcoxon signed rank test was conducted to compare the difference before and after the intervention. The significance level was set at .05 .

\section{Results}

In total, 10 children with cerebral palsy were recruited and two among them dropped out due to academic or personal reasons. Only eight children completed their participation in this study (five boys and three girls). The average age of the subjects was 14 (SD 3.11, range 9-18). As for the types of spasticity of the children with cerebral

Table 1. General Charactgeristics of Subjects

\begin{tabular}{lc}
\hline \multicolumn{1}{c}{ Parameters } & Subjects $(\mathrm{n}=8)$ \\
\hline Gender (male/female) & $5 / 3$ \\
Age (years) & $14 \pm 3.12$ \\
Height & $152.88 \pm 17.21$ \\
Weight & $45.75 \pm 15.23$ \\
Type (hemi/di/quard) & $4 / 3 / 1$ \\
\hline
\end{tabular}

palsy, four had the hemiplegia type, three had diplegia, and one had quadriplegia. The demographic and clinical characteristics of the participating subjects are summarized in Table 1. As for the change in measurement values after the six-week intervention, 10MWT showed a decreased result compared to that before the training, but it was not statistically significant ( $>$.05). On the contrary, TUG and postural sway indicate static balance showed a statistically significant decrease $(\mathrm{p}<.05)$ (Table 2).

In a static balance test using the Good Balance System ${ }^{\circledR}$, the average moving speed statistically significantly decreased in the AP and ML directions $(\mathrm{p}<.05)$, and the mean velocity moment also significantly decreased $(\mathrm{p}<.05)$ (Table 2).

\section{Discussion}

The purpose of this study was to determine the impact of balance training on a three-dimensional balance trainer that provides visual feedback, as well as of left-and-right and up-and-down movements through knee joint movements on the functions of children with spastic cerebral palsy.

The study results show that TUG, except for 10MWT and static balance in postural sway using the Good Balance System ${ }^{\circledR}$, showed a statistically significant improvement after the six-week intervention. That is, balance training on the three-dimensional balance trainer induced

Table 2. The Changes of Parameters after intervention

\begin{tabular}{llllc}
\hline \multicolumn{1}{c}{ variables } & \multicolumn{1}{c}{ pre } & \multicolumn{1}{c}{ post } & \multicolumn{1}{c}{ Z } & p \\
\hline 10MWT & $9.59 \pm 1.29$ & $8.55 \pm .54$ & $-1.680 \mathrm{~b}$ & .093 \\
TUG & $12.22 \pm 1.94$ & $10.6504 \pm 1.63$ & $-2.100 \mathrm{~b}$ & .036 \\
AP Velocity $(\mathrm{mm} / \mathrm{s})$ & $10.24 \pm 3.54$ & $8.4 \pm 3.96$ & $-2.197 \mathrm{~b}$ & .028 \\
ML Verocity $(\mathrm{mm} / \mathrm{s})$ & $10.33 \pm 3.36$ & $8.025 \pm 2.2$ & $-2.240 \mathrm{~b}$ & .025 \\
Velocity moment $(\mathrm{mm} / \mathrm{s} 2)$ & $50.9 \pm 36.30$ & $37.3 \pm 33.83$ & $-2.240 \mathrm{~b}$ & .025 \\
\hline
\end{tabular}

${ }^{*} \mathrm{p}<.05$. 
improvements in dynamic and static balance among the children with cerebral palsy. These results are consistent with the previous study results that reported a significant impact of balance training using visual feedback on balance performance ability (Ledebt et al., 2005; Sihvonen et al., 2004; Walker et al., 2000). Moreover, it is consistent with Sackley and Lincoln (1997), who argued that visual feedback training using the proprioceptive sense is effective in improving symmetric posture and sway (Sackley and Lincoln, 1997). That is, horizontal and vertical movements on a three-dimensional balance trainer simultaneously stimulate the visual, proprioceptive, and vestibular senses, in addition to visual feedback, and this increased sensual integration, which ultimately improved balance ability.

The three-dimensional balance trainer has the features of providing up-and-down vertical movement by moving the knee joint, such as during the sit-to-stand movement. In these functional activities, including sit-to-stand, children with cerebral palsy have more postural sway than normally developed children (Pavão et al., 2015). Sit-to-stand training has a positive impact on the improvement of posture control according to postural sway (Ng, 2010). Cheng et al. (2001) reported that sit-to-stand training applying visual and auditory feedback improved the balance ability of patients who had suffered a stroke (Cheng et al., 2001). Ng (2010) reported that sit-to-stand training has a positive impact on balance performance ability. That is, the results of previous studies using sit-to-stand training are consistent with this study. In this study, it is conjectured that the vertical up-and-down movement on the three-dimensional balance trainer made a positive contribution as a closed kinetic chain exercise to joint stability and caused an increase in the strength of the extensor muscles, such as the quadriceps femoris muscles, which are required for the sit-to-stand movement (Iwasaki et al., 2006). This can be supported by Hu et al. (2013) who proved the effectiveness of sit-to-stand training using a stick to strengthen the extensor muscles of patients who had suffered a stroke. Moreover, Salbach et al. (2004) reported the effectiveness of strengthening the lower limb muscles in improving balance in a study using patients who had suffered a stroke as a sample. Through these previous studies' results, it is conjectured that the enhancement of muscle strength through closed kinetic chain exercises in this study affected the TUG test, including sit-to-stand and static balance in postural sway.

Although the 10MWT results showed diminished time in this study, there was no statistically significant difference. It is conjectured that the intervention period was not long enough for a statistically significant impact on walking, as the abnormal walking posture of the subject group was fixated, as they were the children with cerebral palsy of school age. In a previous study, biofeedback balance training also did not show a significant improvement in walking ability (Barclay-Goddard et al., 2005). We believe that a chronic subject group will require interventions that are more diverse and a longer period to improve their walking ability.

Moreover, the three-dimensional balance trainer is capable of motivating subjects for active movement through diverse games as they move COG left-and-right and up-and-down within BOS. This is consistent with the study results that reported that intervention methods that induce active movement of COG within BOS through motivation induction, such as training devices using games, are effective in improving balance ability (Jelsma et al., 2013).

As such, this study confirmed that applying balance training using the three-dimensional balance trainer, with the features of visual feedback and up-and-down knee joint movement, to children with cerebral palsy has effects on increasing dynamic and static balance, except for gait speed. Previous studies also supported these results. It is conjectured that the three-dimensional balance trainer caused a positive impact on dynamic and static balance ability by integrating the diverse senses through visual feedback, strengthening the extensor muscles through 
up-and-down movements centered at the knee joint, and active COG movements within the BOS through motivation induction among the subjects.

The interpretation of this study results has several limitations. First, future studies will be required that use a larger sample, as it is difficult to generalize the effects of the three-dimensional balance trainer for children with cerebral palsy, as the study subjects included only eight children with cerebral palsy. Second, whether the test accurately measured walking performance ability is unclear due to the emphasis on the fragmentary part of the walking function, as the study suggested 10MWT, which measures time only in the walking function. Third, we could not verify the continuity of the training effects in the long term, as the study did not conduct follow-up after the training term. Finally, the cause of contamination could not be fully removed, as the test was designed without a control group for the removal of variables according to time change. Hence, further studies will have to continue to address the suggested limitations so the effects of the three-dimensional balance trainer for children with cerebral palsy can be more accurately explained.

\section{Conclusion}

The balance ability of the spastic cerebral palsy participants after balance training using a three-dimensional balance trainerwere improved.

This indicates that balance training using the three-dimensional balance trainer, with the features of visual feedback and up-and-down knee joint movement effects on increasing dynamic and static balance.

\section{References}

Ballaz L, Robert M, Parent A, et al. Impaired visually guided weight-shifting ability in children with cerebral palsy. Res Dev Disabil. 2014;35(9):1970-7.

Barclay-Goddard R, Stevenson T, Poluha W, et al. Force platform feedback for standing balance training after stroke. Stroke. 2005;36:412-3.

Bax M, Flodmark O, Tydeman C. From syndrome toward disease. Dev Med Child Neurol. 2007;49(109):39-41.

Bigongiari A, e Souza FDA, Franciulli PM, et al. Anticipatory and compensatory postural adjustments in sitting in children with cerebral palsy. Hum Mov Sci. 2011;30(3):648-57.

Butler C, Darrah J. Effects of neurodevelopmental treatment (NDT) for cerebral palsy: an AACPDM evidence report. Dev Med Child Neurol. 2001;43(11):778-90.

Byun SD, Cho DH, Choi WD, et al. Effects of the balance control training in chronic hemiplegic stroke patients. Brain Neurorehabil. 2012;5(1):32-8.

Carlberg EB, Hadders-Algra M. Postural dysfunction in children with cerebral palsy: some implications for therapeutic guidance. Neural plast. 2005;12(2-3):221-8.

Cheng PT, Wu SH, Liaw MY, et al. Symmetrical body-weight distribution training in stroke patients and its effect on fall prevention. Arch Phys Med Rehabil. 2001;82(12):1650-4.

Chen IC, Cheng PT, Chen CL, et al. Effects of balance training on hemiplegic stroke patients. Chang Gung Med J. 2002;25:583-90

Dewar R, Love S, Johnston LM. Exercise interventions improve postural control in children with cerebral palsy: a systematic review. Dev Med Child Neurol. 2015; 57(6):504-20.

Duarte M, Zatsiorsky VM. Effects of body lean and visual information on the equilibrium maintenance during stance. Exp Brain Res. 2002;146(1):60-9.

Ferdjallah M, Harris GF, Smith P, et al. Analysis of postural control synergies during quiet standing in healthy children and children with cerebral palsy. Clin Biomech. 2002;17(3):203-10. 
Girolami GL, Shiratori T, Aruin AS. Anticipatory postural adjustments in children with hemiplegia and diplegia J Electromyogr Kinesiol. 2011;21(6):988-97.

Ha H, Cho K, Lee W. Reliability of the Good Balance System for Postural Sway Measurement in Poststroke Patients. J Phys Ther Sci. 2014;26(1):121-4.

Hu PT, Lin KH, Lu TW, et al. Effect of a cane on sit-to-stand transfer in subjects with hemiparesis. Am J Phys Med Rehabil. 2013;92(3):191-202.

Imms C. Children with cerebral palsy participate: a review of the literature. Disabil Rehabil. 2008;30(24): 1867-84.

Iwasaki T, Shiba N, Matsuse H, et al. Improvement in knee extension strength through training by means of combined electrical stimulation and voluntary muscle contraction. Tohoku J Exp Med. 2006;209(1):33-40.

Jelsma J, Pronk M, Ferguson G, et al. The effect of the Nintendo Wii Fit on balance control and gross motor function of children with spastic hemiplegic cerebral palsy. Dev Neurorehabil. 2013;16(1):27-37.

Ledebt A, Becher J, Kapper J, et al. Balance training with visual feedback in children with hemiplegic cerebral palsy: effect on stance and gait. Motor Control. 2005;9:459-68.

Lee DH, Choi SJ, Choi HS, et al. Comparison of Visual and Auditory Biofeedback during Sit-to-stand Training for Performance and Balance in Chronic Stroke Patients. J Korean Soc Phys Med. 2015;10(4):59-68.

Lee EJ, Kim JS. The Changes of Gross Motor Function and Balance Ability in Children with Spastic Diplegic Cerebral Palsy by Trunk Muscle Strengthening Exercise : Single Group Repeated Measure Study. JKorean Soc Phys Med. 2011;6(2):189-97.

Lee HM. Effects of Virtual Reality Based Video game and Rehabilitation Exercise on theBalance and Activities of Daily Living of Chronic Stroke Patients. JKorean Soc Phys Med. 2013;8(2):201-7.

Nelson KB. Can we prevent cerebral palsy?. N Engl J Med.
2003;349:1765-9.

Ng S. Balance ability, not muscle strength and exercise endurance, determines the performance of hemiparetic subjects on the timed-sit-to-stand test. Am J Phys Med Rehabil. 2010;89(6):497-504.

Pavão SL, Santos AN, Oliveira AB, et al. Postural control during sit-to-stand movement and its relationship with upright position in children with hemiplegic spastic cerebral palsy and in typically developing children. Braz J Phys Ther. 2015;19(1):18-25.

Podsiadlo D, Richardson S. The timed "Up \& Go": a test of basic functional mobility for frail elderly persons. J Am Geriatr Soc. 1991;39(2):142-8.

Sackley CM, Lincoln NB. Single blind randomized controlled trial of visual feedback after stroke: effects on stance symmetry and function. Disabil Rehabil. 1997;19(12):536-46.

Salbach N, Mayo N, Wood-Dauphinee S, et al. A task-orientated intervention enhances walking distance and speed in the first year post stroke: a randomized controlled trial. Clin rehabil. 2004;18(5):509-19.

Shumway-Cook A, Horak FB. Assessing the influence of sensory interaction on balance suggestion from the field. Phys Ther. 1986;66(10):1548-50.

Shumway-Cook A, Hutchinson S, Kartin D, et al. Effect of balance training on recovery of stability in children with cerebral palsy. Dev Med Child Neurol. 2003;45(9):591-602.

Sihvonen SE, Sipilä S, Era PA. Changes in postural balance in frail elderly women during a 4-week visual feedback training: a randomized controlled trial. Gerontol. 2004;50(2):87-95.

Van Der Heide JC, Begeer C, Fock JM, et al. Postural control during reaching in preterm children with cerebral palsy. Dev Med Child Neurol. 2004;46(4):253-66.

Walker C, Brouwer BJ, Culham EG. Use of visual feedback in retraining balance following acute stroke. Phys Ther. 2000;80(9):886-95. 